\title{
Uniform Rectangular Array Synthesis and Optimization Using Modified Genetic Algorithm
}

\author{
Mercy Sheeba $\mathrm{J}^{\mathrm{a}, 1}$ and S.Deepa ${ }^{\mathrm{b}}$ \\ ${ }^{\mathrm{a}} \mathrm{PGS}$ cholar, ${ }^{\mathrm{b}}$ Professor, \\ ${ }^{a, b}$ Dept of ECE, Panimalar Engineering College, Chennai, India
}

\begin{abstract}
This paper aims to synthesize a uniform rectangular array (URA) which spans beamwidth of $-30^{\circ}$ to $30^{\circ}$ in the azimuthal direction with the interference direction as $40^{\circ}$ in the azimuth plane. In this paper, a Modified Genetic Algorithm is proposed which works to produce a beam pattern with a narrow beamwidth, high directivity and maximized side lobe level (SLL) suppression. The simulation results for the proposed algorithm demonstrates that the synthesized beam pattern for a $16 \times 16$ URA at a frequency of $1 \mathrm{GHz}$ converges to the desired optimum solution producing a maximum SLL suppression of $-30 \mathrm{~dB}$.
\end{abstract}

Keywords. Modified Genetic Algorithm, SLL, URA, array synthesis,optimization algorithm

\section{Introduction}

The most important requirement when synthesizing beam patterns is the suppression of side lobes. The significance of side lobe suppression is underlined by the fact that excessive side lobes result in interference and energy wastage. Pointing nulls in the direction of side lobe arrival, suppresses the side lobe level (SLL) which in turn leads to interference suppression. One major problem to be addressed while synthesizing a phased array is to determine a match between the desired and synthesized array patterns using the most suitable optimization strategy. The specifications to the array synthesizing problem are stated interms of beamwidth and SLL. The Uniform Rectangular Array (URA) is known for its ability to steer the beams in both the azimuthal and elevation planes [2]. It finds its applications in adaptive beamforming, 2-D Esprit and MUSIC algorithms. To procure an optimized beam pattern for the URA, the Modified Genetic Algorithm is proposed which works with the objective of attaining maximum SLL suppression of $-30 \mathrm{~dB}$ in both planes. This paper is organized as follows: The review of different optimization techniques is carried out in Section 2. Section 3 describes the working of the proposed optimization algorithm. The simulated results with its conclusion are presented in Section 4 and 5, respectively.

\section{Related Works}

In Massive MIMO environment, where thousands of antenna elements are involved, it becomes difficult and time consuming for an array architecture to converge to specific

\footnotetext{
${ }^{1}$ Mercy Sheeba J, PG Scholar, Dept of ECE, PanimalarEngineering College, Chennai, India;

E-mail: mercyjawahar7@gmail.com
} 
array patterns. In such situations, to ease the difficulty, optimization techniques are used. These optimization techniques serve to improve the efficiency of the entire array synthesis process by performing automated iterations and evaluates the array patterns over different weights and element positions.Several optimization algorithms have been reviewed in literature. Flower pollination algorithm (FPA) though simple to implement, yielding improved performance, it is more time consuming than Particle Swarm Optimization (PSO) with more number of iterations to converge to an optimum solution [9]. In PSO, all particles move through global search and end with local search at the last generation, whereas in FPA, both global and local search are carried out in each generation making it more time consuming. FPA, however has a faster convergence rate than Ant Colony Optimization (ACO) [7]-[10]. The side lobe level for 10-element equally spaced LAA using FPA algorithm is $-25.32 \mathrm{~dB}$ and for PSO is $-24.62 \mathrm{~dB}$ [8]. The side lobe level for 12-element unequally spaced LAA using FPA algorithm is $-20.764 \mathrm{~dB}$ and for PSO is $-17.90 \mathrm{~dB}$ [8]. For a 10 element linear array, it can be noted from literature [3] that GWO produces a peak SLL of -23.42 $\mathrm{dB}$ which is $10.19 \mathrm{~dB}$ lower to that of conventional array. The three fundamental steps that define the genetic algorithm are evaluation, selection and recombination [4]-[6]. Genetic Algorithm offers several advantages which makes it more commanding than other optimization techniques [6]. The fitness function [11] of Social Group Optimization Algorithm (SGOA) [1] considers both SLL reduction and beamwidth constraints. From literature[11-13], it is stated that for circular array antenna with $\mathrm{N}=$ 30 elements, low SLL of $-15.83 \mathrm{~dB}$ is achieved with the uniform BW of $18.2^{\circ}$ and for $\mathrm{N}=60$ elements, the corresponding $\mathrm{BW}$ is $9.1^{\circ}$ with an SLL of $-7 \mathrm{~dB}$. Using SGOA, the SLL is suppressed to $-18.87 \mathrm{~dB}$.

\section{Proposed OptimizationAlgorithm}

\subsection{Array Synthesis and Optimization Workflow}

The synthesis of array patterns and its optimization workflow is illustrated in Figure 1. The process of determining a best match between the desired and synthesized array patterns involves the following steps:-(i)Observe the initial beam pattern and identify the desired pattern attributes such as narrow beamwidth, lower side lobes etc., (ii)Choose a specific array size and its geometry depending on the desired beamwidth (iii)Develop an objective function to steer the beam pattern in the required direction (iv)Develop constraints for the outputs(for eg: weight and element position) (v)Run theoptimization(vi)Generateanoptimizedpatternandcomparewithdesiredpattern(vii) Repeat process by extending across different frequencies and steering angles.

\subsection{Modified GeneticAlgorithm}

In modified Genetic Algorithm, the elements in the URA can be turned on/off based on the requirements. The modified genetic algorithm works as follows:-

Step 1: An initial population of $\mathrm{P}$ individuals is generated in a random fashion within the variable constraint range.

Step 2: The fitness function is defined and evaluated for each individual candidate. 


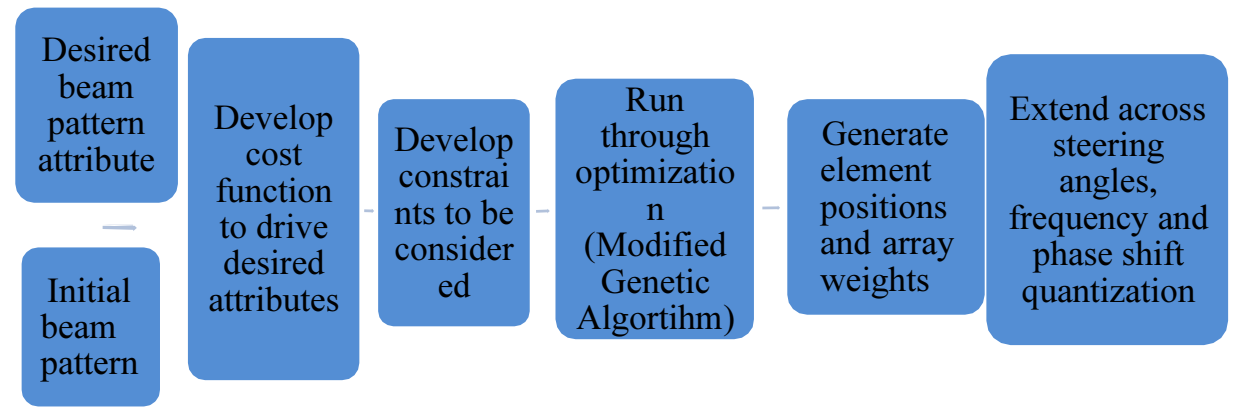

Figure 1. Array Synthesis Workflow for optimizing both weights and antenna position

Step 3: Based on the performance of the individuals according to the specified performance criteria, the population is refined. The individual candidates exhibiting high fitness are placed in the mating section and those with lower performance measure are discarded.

Step 4: The individuals placed in the mating pool involve in mating with the other individual, thereby two individuals mate and then mutate using crossover and mutation processes, respectively. In crossover, two parents mate each other to produce two children. Mutation on the other hand, changes only one variable of a parent and performs a similar process as crossover.

Step 5: In the newly formed generation, the worst individuals are replaced by the best ones in the former generation. The fitness function is then tested for convergence to ensure optimized solutions are obtained. This step makes sure that the best individuals are not lost while moving on to newer generations.

Step 6: The steps 2-5 are repeated until a stopping criterion is met. The criterion can be either achieving a desired solution or based on the number of generations. In this way, the best individual is identified and taken as the optimized solution in synthesizing the array patterns.

\section{Simulation Results}

In this work, as a first step, we have considered the synthesis of a 10-element ULA (Uniform Linear Array) whose scanning region ranges from -30 and 30 degrees with a null placed at 40 degrees. The antenna array synthesis and optimization is carried out in Matlab. The power distribution for the scanned region $-30^{\circ}$ to $30^{\circ}$ azimuthal direction can be seen from Figure 2. The 3D beam pattern (Figure 3) can be more effectively attained with the help of URA. No back lobes are present which means that no energy is radiated in the backward direction. To determine the array size, we set the spacing between the array elements to half its wavelength. In a URA, for half wavelength spacing, the number of elements in a particular direction can be approximated as,

$$
N \approx \frac{2}{\sin \left(\theta_{b}\right)}
$$

where $\theta_{b}$ is the beamwidth along that direction. Using Eq.(1), the aperture size of URA is computedas,

$$
\begin{aligned}
& \text { N_row }=\operatorname{round}(2 / \operatorname{sind}(\operatorname{diff}(\text { elco }))) \\
& \text { N_col }=\operatorname{round}(2 / \operatorname{sind}(\operatorname{diff}(\operatorname{azco})))
\end{aligned}
$$


whereN_row is the number of rows and N_col is the number of columns of URA; elco and azco are the elevation cutoff and azimuthal cutoff, respectively.

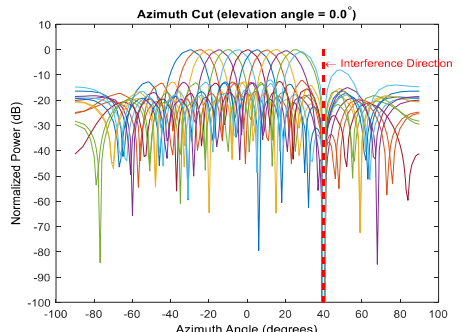

Figure 2. Powerdistributionpattern for look directions $\left(-30^{\circ}\right.$ to $\left.30^{\circ}\right)$ with interference at $40^{\circ}$ in azimuthal plane

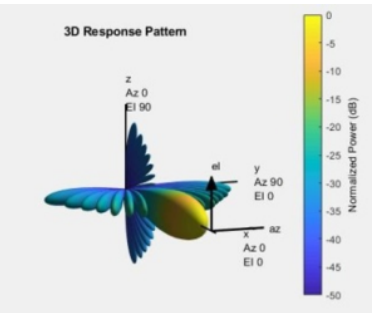

Figure 3. 3D Radiation Pattern of URA

Based on Eqs.(2) and (3), the number of rows and columns are estimated to be 14 and 19 , respectively. The side lobe levels (SLL) of the synthesized beam pattern (Figure 4) is closer to $-12 \mathrm{~dB}$ in both the azimuth and elevation direction and is larger than that of the desired pattern. By applying windowing functions for array synthesis, the SLL isalleviated further to $-25 \mathrm{~dB}$ as in Figure 5 .

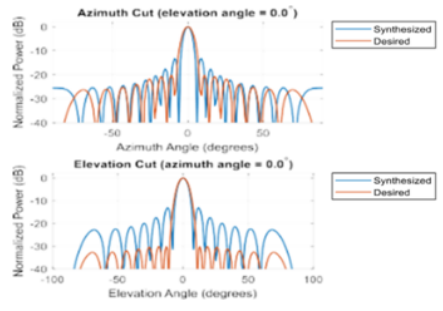

Figure 4. Synthesized and Desired SLL of $14 \times 19$ URA

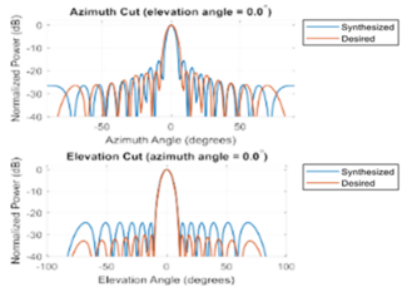

Figure 5. Synthesized and Desired SLL of 14x19 URA on applying windowing operations

To suppress the side lobes furthermore, the number of rows and columns are modified based on trial and error method. A maximum side lobe suppression of $-30 \mathrm{~dB}$ which matches the requirements of the desired pattern (Figure 6) is achieved using Modified Genetic Algorithm. The optimized beam pattern is achieved with a reduction in the number of URA elements. The desired and synthesized 3D pattern of a 16x16 URA and its taper design are displayed in Figure 7.

\section{Conclusion}

The synthesized URA produced anoptimizedbeam pattern using Modified Genetic Algorithm which spanned a beamwidthof- $30^{\circ}$ to $30^{\circ}$ in the azimuthal direction and also places a null at $40^{\circ}$ in the azimuth plane for interference cancellation through side lobe suppression. The synthesized array pattern for a 16x16 URA converged to the desired beam pattern attributes with the use of Modified Genetic Algorithm and 
suppressed the side lobe levels to-30dB.
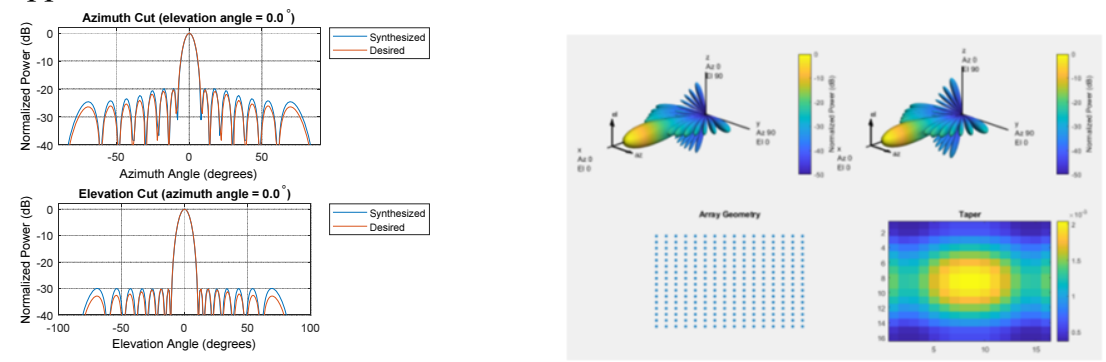

Figure 6. Synthesized and Desired SLL

Figure 7. (a) Desired 3D pattern (b) Synthesized3D 16x16 URA pattern (c) Array Geometry and (d) Taper

\section{References}

[1] Abdul RahimanSheik, Kalva Sri Rama Krishna,Optimal Design and Synthesis of Linear Antenna Array Using Social Group Optimization Technique. in International Journal of Innovative Technology and Exploring Engineering (IJITEE) ISSN: 2278-3075, Volume-8 Issue-7 May,2019

[2] SaswatiGhosh and DebaratiSen, An Inclusive Survey on Array Antenna Designfor Millimeter-Wave Communications/ IEEE Access, June 24,2019

[3] PrernaSaxena and AshwinKothari,Optimal Pattern Synthesis of Linear Antenna Array Using Grey Wolf Optimization Algorithm.in International Journal of Antennas and Propagation,2016.

[4] J.ShahidehKiehbadroudinezhad, Nor KamariahNoordin, A. Sali, and ZamriZainalAbidin.OptimizationOfAn Antenna Array Using Genetic Algorithms.in Astronomical Journal,2014.

[5] G. K. Mahanti, N. Pathaket.al.,.SynthesisOfThinned Linear Antenna Arrays With Fixed Sidelobe Level Using Real-Coded Genetic Algorithm. Progress In Electromagnetics Research,2007.

[6] Stelios C. A. Thomopoulos, Christos N. Capsaliset.al.,.Genetic Algorithms in Antennas and Smart Antennas Design Overview: Two Novel Antenna Systems for Triband GNSS Applications and a Circular Switched Parasitic Array for WiMax Applications Developments with the Use of Genetic Algorithms. International Journal of Antennas and Propagation,2014.

[7] V. V. S. S. S. Chakravarthy, P. S. R. Chowdaryet.al.,.On the Linear Antenna Array Synthesis Techniques for Sum and Difference Patterns Using Flower Pollination Algorithm. Arabian Journal for Science and Engineering, July2017.

[8] Urvinder Singh, RohitSalgotra, Synthesis of linear antenna array using flower pollination algorithm.Neural Computing and Applications, July2016.

[9] PrernaSaxena and Ashwin Kothari .Linear antenna array optimization using flower pollination algorithm.Springer Plus, 2016.

[10] V. S. S. S. ChakravarthyVedula, S. R. ChowdaryPaladugaandM. RaoPrithvi.Synthesis of Circular Array Antenna for Sidelobe Level and Aperture Size Control Using Flower Pollination Algorithm.in International Journal of Antennas andPropagation,2015.

[11] V. V. S. S. Sameer Chakravarthy, P. S. R. Chowdary, Suresh Chandra Satpathyet.al.,.Antenna Array Synthesis Using Social Group Optimization.in Springer Nature, Microelectronics, Electromagnetics and Telecommunications, Lecture Notes in ElectricalEngineering.

[12] A.R.Sheik and K.S.R.Krishna, .Linear Antenna Array Synthesis for Unconstrained and Constrained Nulls Using Social Group Optimization Algorithm. Journal of Scientific and Industrial Research, Sept 2019.

[13] Abdul Rahiman Sheik, Dr.Kalva Sri Rama Krishna, .Constrained synthesis of Linear Antenna Array Synthesis Using Social Group Optimization.in International Journal of Engineering \&Technology(2018). 